\title{
MicroRNA-296 mediated corneal neovascularization in an animal model of corneal burns after alkali exposures
}

\author{
KAI-BAO JI $^{1 *}$, LING LING ${ }^{*}$, QIAN ZHANG ${ }^{2}$, JING-JING CHOU ${ }^{2}$, XIA-LING YANG ${ }^{2}$, \\ ZHI-HONG WANG ${ }^{2}$, LI YIN ${ }^{2}$, SHU-FANG WU ${ }^{2}$ and YI-FENG YU ${ }^{2}$ \\ ${ }^{1}$ Department of Ophthalmology, Affiliated Eye Hospital of Nanchang University, \\ Jiangxi Provincial Key Laboratory for Ophthalmology; ${ }^{2}$ Department of Ophthalmology, The Second \\ Affiliated Hospital of Nanchang University, Nanchang, Jiangxi 330006, P.R. China
}

Received July 17, 2017; Accepted October 30, 2017

DOI: $10.3892 / \mathrm{etm} .2017 .5408$

\begin{abstract}
Alkali burns of the cornea may lead to permanent visual impairment or complete blindness. In the current study, the role of microRNA 296 (miR-296) was explored in mouse corneal neovascularization induced by alkali burns. An alkali burn model in Balb/c mice was developed to study chemical corneal injuries. The expression of the miR-296 gene was measured by reverse-transcription-quantitative polymerase chain reaction. Fibroblast growth factor 23 (FGF23) protein expression was measured by western blot analysis. Possible impacted pathways were analyzed by Kyoto Encyclopedia of Genes and Genomes pathway analysis. miR-296 gene expression was examined following chemical corneal injury and it was demonstrated that different topical eye medications decreased miR-296 gene expression. miR-296 may participate in cytokine-cytokine receptor interaction pathways to influence corneal inflammatory responses. It was also revealed that FGF23 was expressed following chemical corneal injury and that different treatments with topical eye drops decreased its expression. miR-296 is a novel molecular modulator for alkali burns in the mouse cornea.
\end{abstract}

\section{Introduction}

Chemical burns are the second most common cause of burns in China (1). According to a previous retrospective study, ocular severe chemical damage leads to devastating injury and accounts for a small but significant percentage of corneal blindness in China (2), for which may be ascribed to the swiftly developing industrialization in Shanghai (3). The

Correspondence to: Dr Yi-Feng Yu, Department of Ophthalmology, The Second Affiliated Hospital of Nanchang University, 1 Minde Road, Nanchang, Jiangxi 330006, P.R. China

E-mail: 171018170@qq.com

*Contributed equally

Key words: cornea, alkali, miR-296 failure of appropriate corneal repair following severe eye injuries (such as those after chemical burns) often leads to loss of vision. Alkali burns lead to some of the most serious corneal injuries because alkaline chemicals penetrate tissue more quickly than acids to reach the corneal stroma and extirpate ocular tissues with severe inflammatory response $(4,5)$. Once corneal tissues are damaged by severe chemical burn injuries, corneal neovascularization occurs frequently. The mechanism of corneal neovascularization is thought to involve an imbalance of proangiogenic and anti-angiogenic factors $(6,7)$. Even though corneal neovascularization participates in the pathology of corneal alkali burns, the molecular mechanism of these irreversible injuries is unknown.

MicroRNAs (miRNAs) are highly conserved small noncoding RNAs (consisting of 19 to 25 nucleotides) that can regulate gene transcription in certain kinds of animal and human tissues $(8,9)$. Studies have illustrated that numerous of miRNAs are expressed in mammalian eyes and can display distinctly different expression patterns and molecular functions $(10,11)$. Among them, miR-296 was shown to be associated with angiogenic endothelial cells (12) and prostate cancer cells (13), and with upregulated VEGF and downregulated Notch1 following cerebral ischemic injury (14). However, little is known about miR-296 involvement in the pathophysiology of chemical burns of the cornea.

In the current study, we investigated whether miR-296 participates in the pathobiology of alkali burns in cornea. The results of our study can further our understanding of the underlying molecular mechanisms of this type of ocular pathology, and may help provide novel clinical treatments for patients with corneal alkali burns.

\section{Materials and methods}

Animals. Care, use, and treatment of all of the animals utilized in this study were in strict compliance with the Statement for the Use of Animals in Ophthalmic and Vision Research. Male Balb/c mice, 6 to 8 weeks old, were purchased from the Animal Sciences Department of Nanchang University. The mice were maintained on a 12-h light/dark cycle for at least 2 weeks prior to the onset of experiments. All experimental procedures were reviewed and approved by the Ethics Committee for Animal 
Table I. Primers list for mouse miR-296 gene qPCR analysis.

\begin{tabular}{lll}
\hline Genes & Forward primers & Reverse primers \\
\hline U6 & 5'-CTCGCTTCGGCAGCACATATACT-3' & 5'-ACGCTTCACGAATTTGCGTGTC-3' \\
miR-296 & 5'-ACACTCCAGCTGGGAGGGCCCCCCCTCAA-3' & 5'-TGGTGTCGTGGAGTCG-3' \\
\hline
\end{tabular}

miR, microRNA; qPCR, quantitative polymerase chain reaction.

Research at the Second Hospital Affiliated with Nanchang University, Nanchang, China.

Experimental animal model of corneal burns after exposure to alkali. The mice were studied while under general anesthesia induced by intraperitoneal injection of $0.3 \mathrm{ml} / \mathrm{kg}$ chloral hydrate, followed by topical anesthesia of the eye by drops of $0.5 \%$ proparacaine hydrochloride (Alcon, Inc., Fort Worth, TX, USA). A sheet of filter paper (Sigma-Aldrich; Merck KGaA, Darmstadt, Germany) was cut to size in order to provide disk-shaped pieces with diameters of $2 \mathrm{~mm}$. These filter paper disks were presoaked in $1 \mathrm{~N} \mathrm{NaOH}$, and then applied to the center of the anesthetized cornea of the right eye of each mouse for $10 \mathrm{sec}$, followed by immediate eye irrigation with balanced salt solution (Alcon, Inc.), as previously described (15), and the animals were then randomly allocated to 1 of 3 different treatment groups: Levofloxacin eye drops (KB2), levofloxacin and FK506 eye drops (SY1), or levofloxacin and tobramycin dexamethasone eye drops (SY2).

Total RNA sample preparation and quantitative polymerase chain reaction ( $q P C R)$. To quantify miR-296 mRNA expression levels, frozen mice corneal tissues were dissolved in Trizol (Takara Biotechnology Co., Ltd., Dalian, China) and treated 3 times with liquid nitrogen for $5 \mathrm{~min}$ and then a $37^{\circ} \mathrm{C}$ water bath for $5 \mathrm{~min}$. Total RNA was extracted from the tissues according to the manufacturer's guidelines. Purified total RNA $(1 \mu \mathrm{g})$ was then reverse transcribed into cDNA using a RevertAid $^{\mathrm{TM}}$ First Strand cDNA Synthesis kit (Fermentas; Thermo Fisher Scientific, Inc., Pittsburgh, PA, USA). Specific primers (Table I) were synthesized by Invitrogen; Thermo Fisher Scientific, Inc. Real-time PCR was performed using the TransStart Tip Green qPCR SuperMix system (TransGen Biotech, Beijing, China) using the following conditions: Incubation at $95^{\circ} \mathrm{C}$ for $10 \mathrm{~min}$, followed by 40 cycles of $15 \mathrm{sec}$ at $95^{\circ} \mathrm{C}$ and $1 \mathrm{~min}$ at $60^{\circ} \mathrm{C}$. All samples were tested in triplicate. PCR reactions and the mean of the reactions were used for calculating expression levels. All data were collected from the linear range of amplification. Expression levels were normalized for the average of U6 mRNA levels from the same samples.

KEGG pathway enrichment analysis of differentially expressed proteins. Kyoto Encyclopedia of Genes and Genomes (KEGG) is a database for systematic analysis of gene function, linking genome, and higher order information. This resource facilitates the study of gene and expression data as a whole network. Our study was performed using the KEGG database (http://www.genome.jp/kegg/) $(16,17)$ and

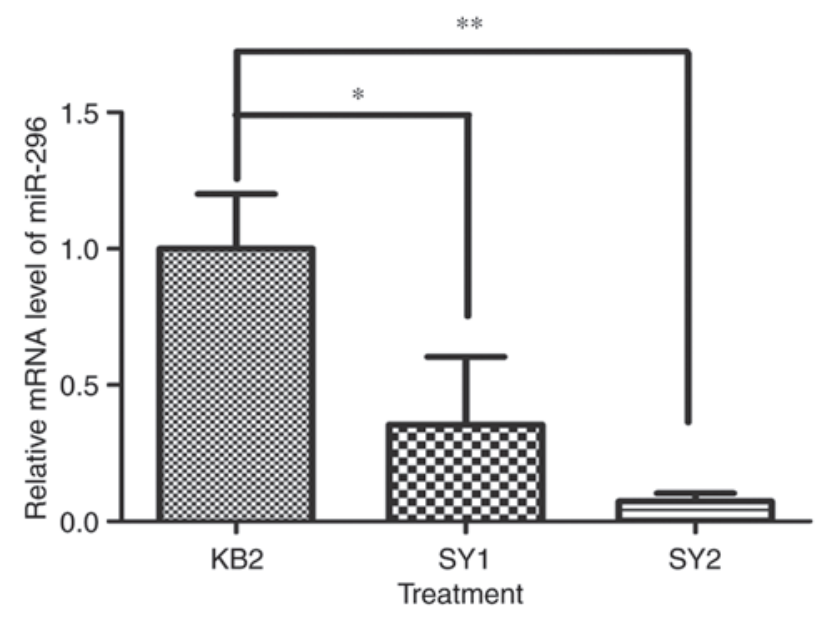

Figure 1. miR-296 mRNA expressed at different levels in different treatment on day 7 after corneal alkali burn. Histogram showing miR-296 mRNA expression level as determined by quantitative polymerase chain reaction. The $y$-axis shows miR-296 expression levels relative to the $\beta$-actin reference gene. Data shown are based on the linear conversion of $\Delta \mathrm{Cq}$ values for each sample $\left(n=3\right.$, error bars denote standard error of the mean; $\left.{ }^{*} \mathrm{P}<0.05,{ }^{* *} \mathrm{P}<0.01\right)$.

Fisher's exact test. We selected the standard as the difference in the number of genes on a term/pathway $\geq 5, \mathrm{P}<0.05$. The term/pathway was drawn based on the enrichment factor value from the descending order of size, taking into account the first 12 results.

Western blot analysis. To quantify FGF23 protein expression levels, frozen mouse corneal tissues were dissolved in tissue RIPA lysis buffer containing $50 \mathrm{mM}$ Tris- $\mathrm{HCl}$ (pH 7.4); Igepal $1 \%(\mathrm{w} / \mathrm{v}) ; 0.25 \%$ (w/v) Na-deoxy cholate; $1 \mathrm{mM}$ EDTA, $150 \mathrm{mM} \mathrm{NaCl} ; 1 \mu \mathrm{g} / \mathrm{ml}$ each of protease inhibitors aprotinin, leupeptin and pepstatin; $1 \mathrm{mM} \mathrm{Na} \mathrm{VO}_{4}$; and $1 \mathrm{mM}$ NaF. Following ultrasonic dispersion, the samples were centrifuged at $12,000 \mathrm{x}$ g for $10 \mathrm{~min}$ at $4^{\circ} \mathrm{C}$. The supernatant was then collected, and total protein concentration was determined using the Pierce BCA Protein Assay kit (Thermo Fisher Scientific, Inc.). Protein samples of tissues were loaded with an equal volume of $2 \mathrm{X}$ SDS-PAGE loading buffer (100 mM Tris-HCl, pH 6.8; 4\% SDS; $20 \%$ glycerol; $0.2 \%$ bromphenol blue and $0.14 \% \beta$-mercaptoethanol) and boiled (for $5 \mathrm{~min}$ at $100^{\circ} \mathrm{C}$ ), and the protein samples were stored at $-20^{\circ} \mathrm{C}$ until use. For immunoblotting, $20 \mu \mathrm{g}$ of the total protein samples were resolved on $12 \%$ SDS-PAGE, at a constant voltage of $100 \mathrm{~V}$ for $2 \mathrm{~h}$. The proteins resolved in the gel were then transferred to nitrocellulose membranes (EMD Millipore, Billerica, MA, USA) using Tris/glycine buffer, $\mathrm{pH} 8.3$ (25 mM Tris base, $192 \mathrm{mM}$ glycine, $0.1 \% \mathrm{w} / \mathrm{v}$ 


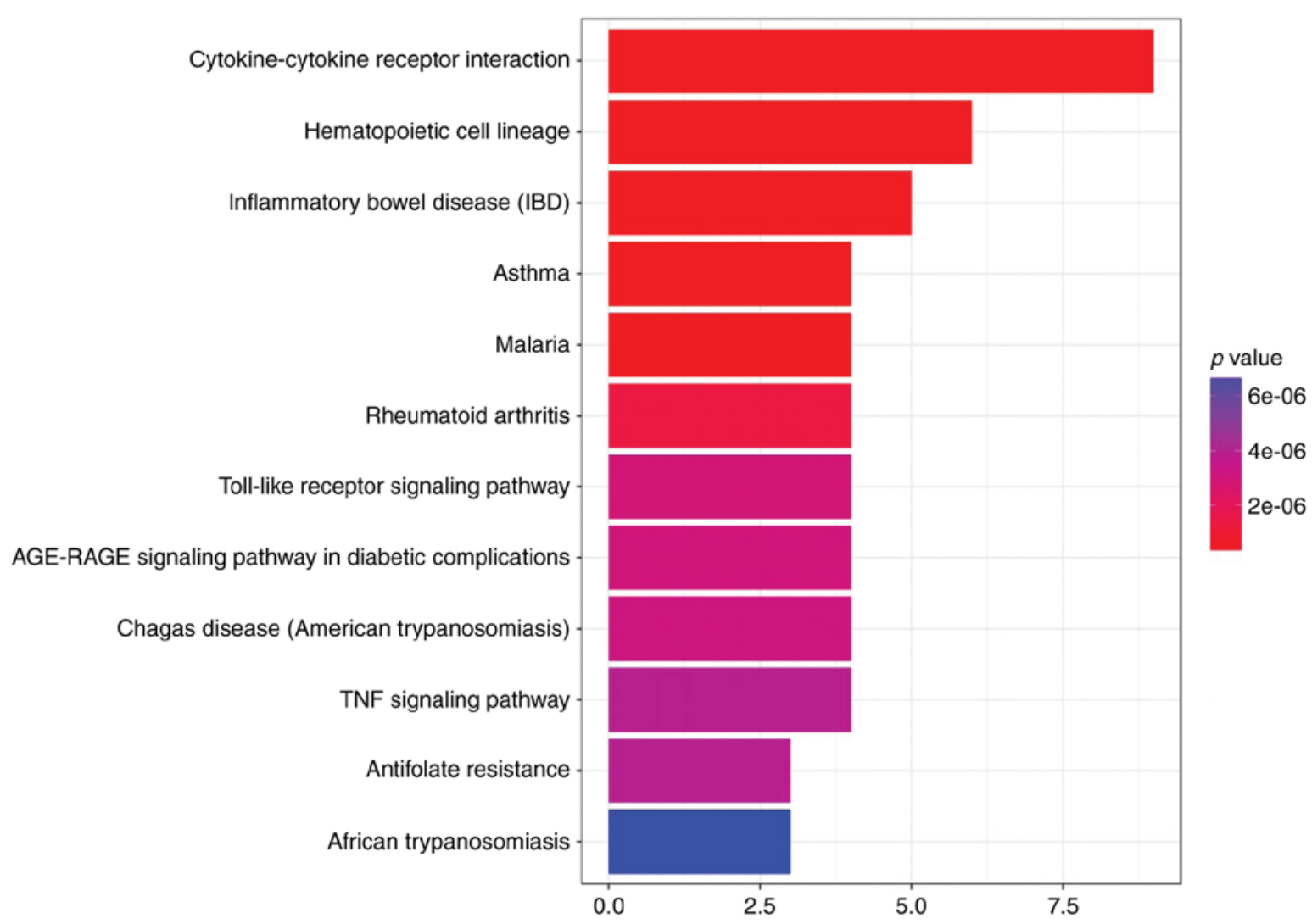

Figure 2. Probable pathway clustering analysis of miR-296 gene in comparison SY1 with KB2 according to KEGG pathway analysis.

SDS, $20 \% \mathrm{v} / \mathrm{v}$ methanol) at 250 to $300 \mathrm{~mA}$ for $3 \mathrm{~h}$. The membranes were then blocked for $1 \mathrm{~h}$ in non-fat powdered milk $(5 \%)$ in TBST washing buffer $(10 \mathrm{mM}$ Tris, $150 \mathrm{mM}$ $\mathrm{NaCl}, 0.1 \%$ Tween-20), and incubated with the primary antibody of interest overnight at $4{ }^{\circ} \mathrm{C}$. Rabbit polyclonal anti-FGF23 (1:200; Wuhan Boster Biological Technology, Ltd., Wuhan, China) were used, and the membranes were then washed in TBS-Tween-20 three times over $15 \mathrm{~min}$ and incubated with HRP-conjugated goat anti-rabbit IgG (1:2,000; ZSGB-BIO; OriGene Technologies, Inc., Beijing, China) for $1 \mathrm{~h}$ at ambient temperature and washed in TBST washing buffer as above. Immunoreactivity was visualized using film exposure. To allow normalization for minor variances in protein loading between sample lanes, the blots were re-probed for anti- $\beta$-actin $(1: 1,000$; ZSGB-BIO; OriGene Technologies, Inc.). ImageJ software (National Institutes of Health, Bethesda, MD, USA) was used to analyze immunoblotting images. Optical densities of all bands were measured with the image background subtracted to give the final densitometry values.

Statistical analysis. The data are presented as mean values \pm standard errors. The Kolmogorov-Smirnov test was used to check the normal distribution of the datasets. The one-way analysis of variance (ANOVA) for measurements was performed using GraphPad Prism version 5.0 (GraphPad Software, Inc., La Jolla, CA, USA), with Turkey's post hoc test for multiple comparisons where appropriate. $\mathrm{P}<0.05$ was considered to indicate a statistically significant difference. All experiments were repeated at least three times.

\section{Results}

In this study of corneal chemical burn wounds, we found that the expression levels of miR-296 mRNA varied significantly in the different treatment groups of our mouse eye model. The results revealed that the data were normally distributed $(\mathrm{P}>0.05)$. There was a statistically significant difference between miR-296 mRNA with KB2, SY1 and SY2, as determined by ANOVA $(\mathrm{P}<0.01)$. Turkey's post-hoc test revealed the level of miR-296 mRNA expressed was significantly decreased in SY1 and SY2 compared with KB2 (Fig. 1, $\mathrm{P}<0.05, \mathrm{P}<0.01)$. No significant difference was seen between SY1 and SY2. This result suggested that the miR-296 gene may be involved in the regulation of the pathophysiology of the alkali-burned cornea, and levofloxacin and tobramycin dexamethasone eye drops (SY2) or levofloxacin eye drops and FK506 eye drops (SY1) were both better than the single-ingredient levofloxacin eye drops for treatment of the alkali-burned cornea. Because we found that miR-296 was actively involved in the reaction to alkali burns, we wondered about the mechanism by which it affects the chemical injury in mouse cornea. Therefore, we evaluated the likely pathways that it might affect utilizing a KEGG pathway analysis (Figs. 2 and 3).

We found that FGF23 protein can be expressed differently after different treatments of the corneal alkali burn model. The results revealed that the data were normally distributed $(\mathrm{P}>0.05)$. There was a statistically significant difference between FGF23 protein with KB2, SY1 and SY2, as determined by ANOVA $(\mathrm{P}<0.01)$. Turkey's post-hoc test 


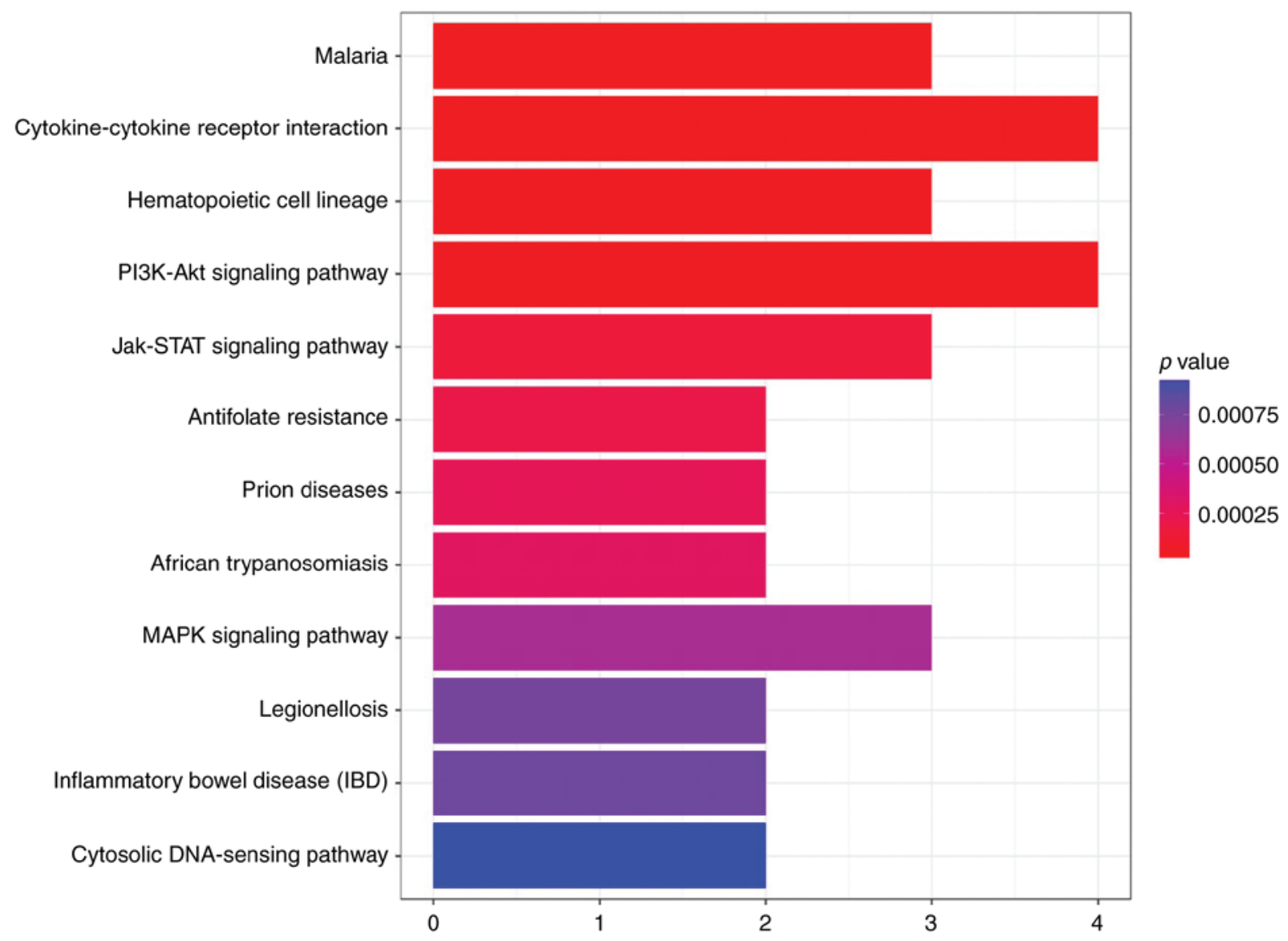

Figure 3. Probable pathway clustering analysis of miR-296 gene in comparison SY2 with KB2 according to KEGG pathway analysis.

B

A

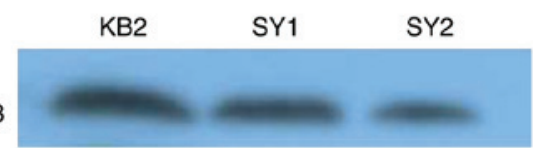

$\beta$-actin

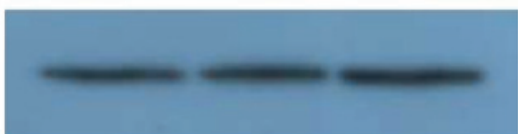

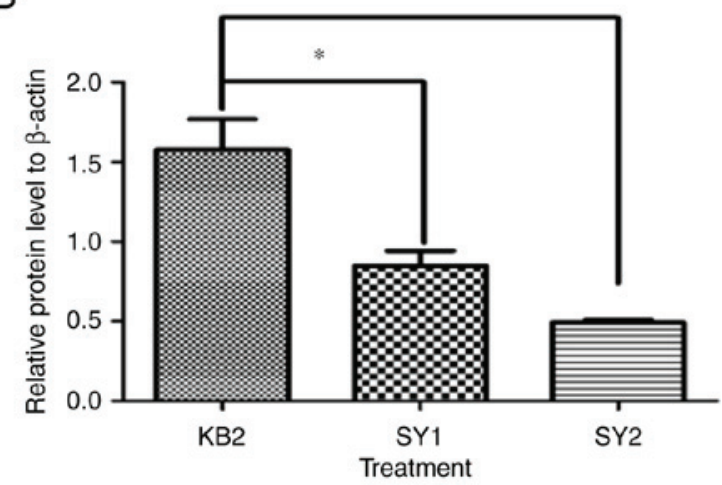

Figure 4. FGF23 protein expressed at different levels in different treatment on day 7 after corneal alkali burn. Representative western blots showing (A) FGF23 protein level in the mouse cornea. The corresponding $\beta$-actin protein level for the same blot is shown, and serves as a protein loading control. The $y$-axis shows (B) the relative FGF23 protein expression level relative to the $\beta$-actin loading control ( $\mathrm{n}=6$, error bars denote standard error of the mean; " $\mathrm{P}<0.05$, * $\mathrm{P}<0.01$ ).
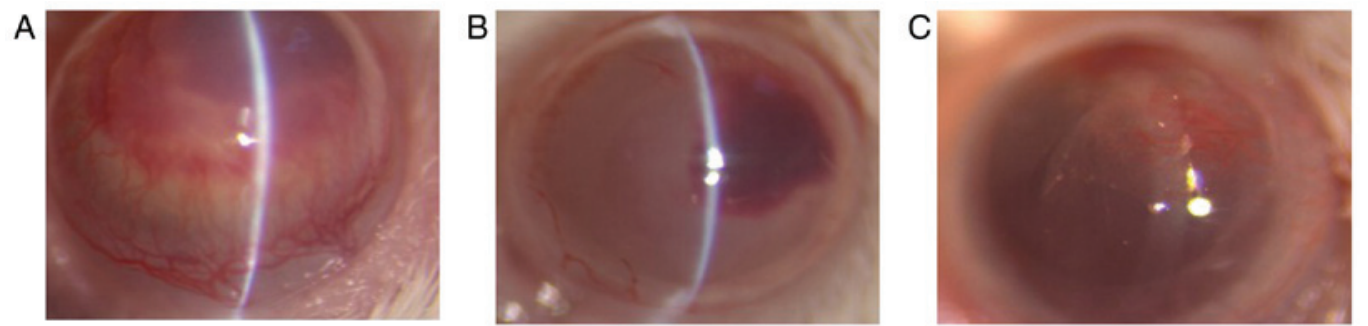

Figure 5. Corneal neovascularization in different treatment on day 7 after corneal alkali burn. (A) Corneal neovascularization was treated with levofloxacin eye drops (KB2), (B) corneal neovascularization was treated with levofloxacin and FK506 eye drops (SY1), (C) corneal neovascularization was treated with levofloxacin and tobramycin dexamethasone eye drops (SY2). 
indicated the level of FGF23 protein, in comparison with KB2, was significantly decreased in SY1 and SY2 (Fig. 4, $\mathrm{P}<0.05, \mathrm{P}<0.01)$. Furthermore, we found that SY2 exhibited lower FGF23 protein expression than SY1, but not significant (Fig. 4, P>0.05). This result demonstrated that FGF23 factors may be involved in the regulation of the burned cornea, and levofloxacin and tobramycin dexamethasone eye drops (SY2) were better than levofloxacin eye drops and FK506 eye drops (SY1) or levofloxacin eye drops (KB2) to modulate the damage in alkali burned cornea. Corneal neovascularization was treatment-dependent with the different eye drops on day 7 after corneal alkali burns. Although all mice developed corneal neovascularization after corneal alkali burn, the SY2 exhibited lower corneal neovascularization area compared to SY1 or KB2 (Fig. 5).

\section{Discussion}

Based on the present study, we report for the first time that the miR-296 gene is expressed during the transient inflammatory responses that occur in mouse cornea after alkali burns. More importantly, we found that levofloxacin and tobramycin dexamethasone eye drops (SY2) or levofloxacin eye drops and FK506 eye drops (SY1) were better than levofloxacin eye drops alone to modulate miR-296 gene activity after alkali burns in the mouse eye.

The advantages of employing knowledge base-driven pathway analysis are becoming more and more appreciated by many investigators in medical research, because it can reduce the complexity of functional analysis by grouping thousands of genes into just several hundred pathways. Such analysis can also increase the explanatory power of experimental results by identifying active pathways in different conditions (18). KEGG is an integrated database that is helpful for biological interpretation of genome sequences and other high-throughput data $(19,20)$. In this study, we identified for the first time a candidate for the most important pathway involved in the miR-296 gene-related inflammatory responses in the mouse cornea after alkali burns in the light of KEGG pathway. In addition, we concluded that the most likely overlap pathway was a cytokine-cytokine receptor interaction pathway. Then, we examined the FGF23 protein that participates in the mouse cornea alkali burn and found that SY2 was better than the other formulations (SY1 or KB2) to ameliorate the effects of alkali on the mouse cornea. Corneal neovascularization (CNV) is playing a critical aspect in the pathophysiology of corneal alkali burns. Multistage and distinct molecules contribute to CNV. In corneal diseases, a number of cytokines and growth factors are upregulated and induce infiltration of neutrophils, macrophages, and lymphocytes (21). CNV is also directly related to Macrophages infiltration (22). The current treatments for CNV include corticosteroids and anti-VEGF (22). Dexamethasone, a synthetic corticosteroid, is widely used as a potent anti-inflammatory drug in various diseases including corneal angiogenesis (23). Dexamethasone can not only inhibit IL-1 $\beta$-induced angiogenesis via blocking NF- $\kappa \mathrm{B}$ signaling, but also partially inhibit VEGF-A (24). Dexamethasone has a large therapeutic window. FK-506 (Tacrolimus), an immunosuppressive neutral macrolide, is isolated from streptomyces tsukubaensis (25). A study pointed out FK-506 can suppress
VEGF in a rabbit CNV model (22). At present, we firstly compared SY2 with SY1, and we concluded that SY2 is SY2 was better to ameliorate the effects of alkali on the mouse cornea. We also found that the IL6 protein was not expressed in this experimental model. Corneal neovascularization was decreased, but we did not quantify it on day 7 after the alkali burn. Thus, in subsequent research, we aim to investigate the mechanism of miR-296-mediated corneal neovascularization in the mouse model of alkali burns.

Taken together, we investigated the miR-296 gene and a possible pathway involved in the transient inflammatory responses of mouse cornea following experimental trauma from alkali exposure. The insights from this study may lead to new therapeutic strategies for treatment corneal alkali burns in the clinical setting.

\section{Acknowledgements}

This study was supported by Natural Science Foundation of Jiangxi (grant no. 20161BAB205270).

\section{References}

1. Xie Y, Tan Y and Tang S: Epidemiology of 377 patients with chemical burns in Guangdong province. Burns 30: 569-572, 2004.

2. Zhang $\mathrm{C}$ and $\mathrm{Xu} \mathrm{J}$ : Indications for penetrating keratoplasty in East China, 1994-2003. Graefes Arch Clin Exp Ophthalmol 243: 1005-1009, 2005

3. Hong J, Qiu T, Wei A, Sun X and Xu J: Clinical characteristics and visual outcome of severe ocular chemical injuries in Shanghai. Ophthalmology 117: 2268-2272, 2010.

4. Saika S, Kobata S, Hashizume N, Okada Y and Yamanaka O Epithelial basement membrane in alkali-burned corneas in rats. Immunohistochemical study. Cornea 12: 383-390, 1993.

5. Ishizaki M,Zhu G, Haseba T, Shafer SS and Kao WW: Expression of collagen I, smooth muscle alpha-actin, and vimentin during the healing of alkali-burned and lacerated corneas. Invest Ophthalmol Vis Sci 34: 3320-3328, 1993.

6. Pandya NM, Dhalla NS and Santani DD: Angiogenesis-a new target for future therapy. Vascul Pharmacol 44:265-274, 2006.

7. de Groot H, Schmit-Eilenberger V, Kirchhof J and Augustin AJ: Angiostatic and angiogenic factors. Dev Ophthalmol 46: 1-3, 2010.

8. Ambros V: The functions of animal microRNAs. Nature 431: 350-355, 2004.

9. Ranganathan K and Sivasankar V: MicroRNAs-Biology and clinical applications. J Oral Maxillofac Pathol 18: 229-234, 2014.

10. Ryan DG, Oliveira-Fernandes M and Lavker RM: MicroRNAs of the mammalian eye display distinct and overlapping tissue specificity. Mol Vis 12: 1175-1184, 2006.

11. Karali M, Peluso I, Gennarino VA, Bilio M, Verde R, Lago G, Dollé P and Banfi S: miRNeye: A microRNA expression atlas of the mouse eye. BMC Genomics 11: 715, 2010.

12. Würdinger T, Tannous BA, Saydam O, Skog J, Grau S, Soutschek J, Weissleder R, Breakefield XO and Krichevsky AM: miR-296 regulates growth factor receptor overexpression in angiogenic endothelial cells. Cancer Cell 14: 382-393, 2008.

13. Wei JJ, Wu X, Peng Y, Shi G, Basturk O, Yang X, Daniels G, Osman I, Ouyang J, Hernando E, et al: Regulation of HMGA1 expression by microRNA-296 affects prostate cancer growth and invasion. Clin Cancer Res 17: 1297-1305, 2011.

14. Feng J, Huang T, Huang Q, Chen H, Li Y, He W, Wang GB, Zhang L, Xia J, Zhang $\mathrm{N}$ and Liu Y: Pro-angiogenic microRNA-296 upregulates vascular endothelial growth factor and downregulates Notch1 following cerebral ischemic injury. Mol Med Rep 12: 8141-8147, 2015.

15. Bian F, Pelegrino FS, Henriksson JT, Pflugfelder SC, Volpe EA, Li DQ and de Paiva CS: Differential effects of dexamethasone and doxycycline on inflammation and MMP production in alkali-burned corneas associated with dry eye. Ocul Surf 14: 242-254, 2016. 
16. Draghici S, Khatri P, Tarca AL, Amin K, Done A, Voichita C, Georgescu C and Romero R: A systems biology approach for pathway level analysis. Genome Res 17: 1537-1545, 2007.

17. Kanehisa M, Goto S, Kawashima S, Okuno Y and Hattori M: The KEGG resource for deciphering the genome. Nucleic Acids Res 32 (Database issue): D277-D280, 2004.

18. Du J, Li M, Yuan Z, Guo M, Song J, Xie X and Chen Y: A decision analysis model for KEGG pathway analysis. BMC Bioinformatics 17: 407, 2016.

19. Kanehisa M, Sato Y, Kawashima M, Furumichi M and Tanabe M: KEGG as a reference resource for gene and protein annotation. Nucleic Acids Res 44: D457-D462, 2016.

20. Khatri P, Sirota M and Butte AJ: Ten years of pathway analysis: Current approaches and outstanding challenges. PLoS Comput Biol 8: e1002375, 2012.

21. Wilson SE, Mohan RR, Mohan RR, Ambrósio R Jr, Hong J and Lee J: The corneal wound healing response: Cytokine-mediated interaction of the epithelium, stroma, and inflammatory cells. Prog Retin Eye Res 20: 625-637, 2001.

22. Park JH, Joo CK and Chung SK: Comparative study of tacrolimus and bevacizumab on corneal neovascularization in rabbits. Cornea 34: 449-455, 2015.
23. Nakao S, Hata Y, Miura M, Noda K, Kimura YN, Kawahara S, Kita T, Hisatomi T, Nakazawa T, Jin Y, et al: Dexamethasone inhibits interleukin-1beta-induced corneal neovascularization: Role of nuclear factor-kappaB-activated stromal cells in inflammatory angiogenesis. Am J Pathol 171: 1058-1065, 2007.

24. Nakao S, Zandi S, Lara-Castillo N, Taher M, Ishibashi T and Hafezi-Moghadam A: Larger therapeutic window for steroid versus VEGF-A inhibitor in inflammatory angiogenesis: Surprisingly similar impact on leukocyte infiltration. Invest Ophthalmol Vis Sci 53: 3296-3302, 2012.

25. Yuan J, Zhai JJ, Chen JQ, Ye CT and Zhou SY: Preparation of $0.05 \%$ FK506 suspension eyedrops and its pharmacokinetics after topical ocular administration. J Ocul Pharmacol Ther 25: 345-350, 2009.

This work is licensed under a Creative Commons Attribution-NonCommercial-NoDerivatives 4.0 International (CC BY-NC-ND 4.0) License. 